\title{
Attachment, perceived social support and mental health problems in women with primary infertility
}

\author{
Sadia Saleem ${ }^{1}$, Namra S. Qureshi ${ }^{1 *}$, Zahid Mahmood ${ }^{2}$
}

\begin{abstract}
${ }^{1}$ Department of Psychology, College of Humanities and Social Sciences, George Mason University, Fairfax, VA, USA
${ }^{2}$ Institute of Clinical Psychology, University of Management and Technology, Lahore, Punjab, Pakistan
\end{abstract}

Received: 04 April 2019

Accepted: 08 May 2019

\section{*Correspondence:}

Dr. Namra S. Qureshi,

E-mail: nquresh6@gmu.edu

Copyright: (C) the author(s), publisher and licensee Medip Academy. This is an open-access article distributed under the terms of the Creative Commons Attribution Non-Commercial License, which permits unrestricted non-commercial use, distribution, and reproduction in any medium, provided the original work is properly cited.

\begin{abstract}
Background: Infertility is one of the fastest growing concerns when it comes to reproductive health and most often, women get the blame. Consequently, females suffer from major psycho-social and emotional problems that may lead to serious mental health concerns.

Methods: To fill the gap in literature, a cross-sectional research design was used to measure the attachment styles with spouse, perceived social support, and predict mental health problems in women attending infertility clinics with ages ranging from 19-45 (M 27.21, SD 4.79). Adult Attachment Questionnaire, Multidimensional Perceived Social Support, and Depression Anxiety Stress Scale were used among experimental subjects selected through purposive sampling technique.

Results: About $32 \%$ women reported themselves as secure, $49 \%$ as ambivalent, and $19 \%$ as avoidant in their attachment style with spouse. The results revealed that a significant negative correlation exists between perceived social support and mental health problems among women with infertility. Moreover, women who identify their attachment pattern as Ambivalent perceive less social support and experience more mental health problems.

Conclusions: Education is one of the strongest predictors of how likely infertility is to cause mental health issues while Attachment style is another strong indicator since infertile women with secure attachment pattern have fewer mental health problems. However, the sample size was modest to make any wide-scale assumptions, so further trials with larger participant pools must be performed. Additionally, future studies should include both rural and urban samples with different psychological variables to find the similarities and differences between various groups of people with diverse backgrounds.
\end{abstract}

Keywords: Attachment style, Culture, Infertility, Mental health

\section{INTRODUCTION}

Infertility is a growing concern across the world and approximately $8-12 \%$ of the couples suffer from infertility across the globe, with highest rates seen in South Asia. ${ }^{1}$ It is also estimated that 01 in every 10 couples in the world experiences infertility. There are two types of infertility seen among women: primary or secondary. $^{2}$ A woman is considered infertile when she cannot conceive after twelve or more months of consistent and unprotected sexual intercourse. ${ }^{3}$ Primary infertility is a failure to conceive after more than one year of regular intercourse whereas secondary is the inability to conceive the second time after the first successful conception. ${ }^{4}$

Although often underestimated, infertility is a critical lifetime crisis that has a catastrophic impact on 
psychosocial as well as emotional functioning of an individual. It is deemed a social stigma in many societies that ultimately becomes an emotional burden coupled with deep seeded guilt and trauma. Infertility also leads to poor marital satisfaction, marital discord, and conflict. ${ }^{5}$ In collectivistic cultures characteristic of many third world countries, the purpose of marriage is only truly met when a couple starts a family life by having children as it is considered a source of pride for the family. ${ }^{6}$ The family sees the new born child as a symbol to keep their ancestry alive and someone who will pass on family name and traditions to the next generation. On the other hand, infertility is a social stigma that brings disgrace to the family. $^{7}$

The health conditions in many countries around the globe are far from ideal. Moreover, infertility treatment is very costly. Due to widespread illiteracy and lack of awareness, women are usually held responsible for the inability to conceive and consequently, they experience greater infertility-related serious psychosocial issues, including physical abuse, social isolation, and a constant threat of husband's second marriage. ${ }^{8,9}$ Increased levels of stress and societal pressure can further hinder the ability to conceive, prolonging infertility.

Infertility is a grave concern associated with many negative consequences ranging from emotional and psychological problems to social stigmas, especially for women. The stress of infertility further increases when childlessness becomes a social stigma and the society starts isolating those women. As a result, this increases the risk of acquiring advanced and chronic mental health problems that disrupt normal functioning of an individual. In addition, women who are undergoing infertility treatment tend to experience higher stress and anxiety levels.

Attachment theory has become one of the most influential frameworks to understand the innate need of human beings to develop emotional bonding with the caregiver and a template for future interpersonal relationships. ${ }^{10}$ Attachment is a very important characteristic of adulthood, especially in romantic and marital relationships, and a positive association between marital success and secure attachment style and has also been reported. ${ }^{11,12}$ In the past studies that explored the association between attachment styles and distress among infertile couples, it was found that secure attachment results in greater well-being whereas insecure, avoidant, and anxious attachment styles are associated with high distress. ${ }^{13,14}$ Although these studies demonstrate a link between attachment styles and suffering associated with infertility, little focus is placed on a possible underlying connection between attachment styles and resulting mental health disorders in women with primary infertility.

Social support is seen as a protective measure against the adversities of life and is usually defined as a perception of accessible source of caring attitude in stressful situations. ${ }^{15}$ The connection between perceived social support and the ability to cope with adverse life events has been cited in past studies. ${ }^{16}$ In regards to infertility, the ability to share and openly reveal emotional burden to their social group and obtain social support from significant others, friends, and family becomes a mean of effective coping measure in handling stress. It could also reduce the chances of mental health problems. ${ }^{17-19}$

Past research has also shown that a person who experiences infertility tends to suffer from anxiety, depression, grief, negative self-image, low self-esteem, isolation, suicidal ideation, and decreased marital satisfaction. ${ }^{20,21}$ It is noteworthy that although both partners are affected by this chronic health condition, women are a victim of more consequential mental health problems than men. ${ }^{22}$ There are numerous possible reasons behind increased depression and nervousness in women with infertility, explicitly the perception of loss of motherhood and womanhood, greater self-blame, negative self-image, and perceived social pressure..$^{5}$ This is due to the existing belief in many conventional societies that the inability to reproduce is solely due to defects in females and only they are to be held responsible for it.

Volgsten, Svanberg, and Olsson summarized that infertility, as an emotional, social, and psychological stress, results into four emotional reactions: initial phase with experience of shock and rejection; this reactive phase marked by anger, frustration, guilt, anger, misery, and hopelessness; phase leading to acceptance; and lastly, the resolving phase of coping, adjusting, and planning for future. ${ }^{23}$ There are countless personal, marital, social, and cultural factors that determine the intensity and severity of emotional responses to infertility. ${ }^{5}$

Although earlier studies suggest that women suffering from infertility also undergo social and psychological problems, no clear connections have been found. This research study aims to identify the predictive relationship among attachment styles, perceived social support, and mental health problems with primary infertility undergoing medical treatment. It is hypothesized that attachment style and social support would have an impact on psychological health of women suffering with infertility.

\section{Research Questions}

- How attachment style affects the perceived social support and mental health of women with infertility?

- What factors contribute to psychological and mental well-being of a female?

- What correlation exists between different sociodemographics of infertile patients and how they contribute to mental health disorders? 


\section{METHODS}

The research data was collected in the city of Lahore (the second largest city of Pakistan) using questionnaires. There are two parallel health care facilities running in Pakistan: private and government-based. The government health centers are inexpensive, thus readily approachable by everyone. Contrarily, private health care facilities are very costly, so only individuals with high socio-economic status can access and benefit from them. The participants of the current study were recruited from both government and private hospitals, thus being representative of people with all socioeconomic backgrounds.

\section{Inclusion criteria/participants}

The study sample comprised of 169 women attending infertility clinics for treatment of primary fertility with ages ranging from 19 to 45 years (M 27.21, SD 4.79). To be included in the study, the females had to be married for at least one year without being able to conceive in that year. The participants were recruited using purposive sampling technique from infertility clinics in government hospitals of Lahore, Pakistan.

\section{Exclusion criteria}

Data was cleaned to eliminate incomplete surveys in which participants had not answered all questions. After cleaning, data was digitized to perform the statistical analysis.

The research data was collected via two parallel health care facilities: private and government-based. The government health centers data was collected in 3 months timeframe between October 2016 to January 2017. It took more time to gather data from private clinic due to approvals.

\section{Measures}

\section{Demographic sheet}

A brief demographic sheet was developed to obtain basic information from the participants, including age, years of marriage, years of treatment, and the structure of family.

\section{Adult attachment questionnaire $(A A Q)$}

A 3-item questionnaire was developed to measures three distinct attachment styles: Secure, Anxious/ Ambivalent, and Avoidant attachment.

\section{The Multidimensional Scale of Perceived Social Support (MSPSS)}

It is a scale that evaluates the adequacy of an individual's perceived social support from friends, family, and significant others. ${ }^{24}$ It is composed of 12 items that are rated on a 07 -point Likert scale from 01 as very strongly disagreed to 07 as very strongly agreed. A person can score between 12-84, while higher scores show better perceived social support.

\section{Depression Anxiety Stress Scale (DASS)}

It is a self-report scale consisting of 21 factoes that measures anxiety, depression, and stress on a 4-point scale-Never, Sometimes, Often, and Almost Always. ${ }^{25}$

\section{Procedure}

The ethical consent for the current study was acquired from the Institution Ethical Committee, Institute of Clinical Psychology of University of Management and Technology, Lahore. The in-person recruitment process was used with the support of nursing staff and gynaecologists who referred the participants before or after their clinical consultations. All the participants who met the inclusion criteria were asked if they would be interested in taking part in the current research, and agreed participants were given the detailed description about the aim and objectives of the study. Prior to participation, research participants were given the consent form which comprised of the assurance of confidentiality, anonymity, and the right to withdraw. Few research participants were illiterate, therefore a research coordinator read out all the instructions for them. After obtaining the consent, participants were given the research protocol to complete.

\section{Statistical analysis}

The design of study or statistical analysis is basically a simultaneously study of several processes in one study instead creating a separate study for each thus producing greater understanding. The normal practice when investigating the effects of factors is through calculating mean and standard deviation but it fails to calculate the interaction between different factors. Thus, a linear regression modelling technique is used and conducted to determine whether interaction between different factors is present and not. It also helps us to know what factors greatly affects the dependent variable which is the factor being determined.

\section{RESULTS}

Table 1 summarizes the demographic information of every survey patient/respondent. Survey asked questions about patients education level, age, duration of marriage and most importantly family system type and attachment style which became the basis of this research study.

Table 2 gives the mean and standard deviation to explain the spread of values in the survey. It also gives an indication of how much the survey respondents deviate from the normal, which the mean. Table 3 indicates a significant inverse (negative) relationship between 
perceived social support and mental health problems in women with infertility.

Table 1: Demographic characteristics of participant $(\mathrm{N}=169)$.

\begin{tabular}{|lc|}
\hline Demographic variables & $\%$ \\
\hline Participants' education level & 22 \\
\hline Illiterate & 41 \\
\hline High school or below & 37 \\
\hline College or above & \\
\hline Age & 48 \\
\hline $19-28$ & 52 \\
\hline 29 or above & \\
\hline Duration of marriage & 47 \\
\hline $1-5$ years & 34 \\
\hline $6-10$ & 19 \\
\hline$>10$ years & \\
\hline Family system & 43 \\
\hline Nuclear & 57 \\
\hline Joint & \\
\hline Attachment styles & 34 \\
\hline Secure & 47 \\
\hline Ambivalent & 19 \\
\hline Avoidant
\end{tabular}

Table 2: Means and standard deviations of years of age, years of marriage, and duration of treatment of the participants.

\begin{tabular}{|lll|}
\hline Years & M & SD \\
\hline Age & 27.21 & 4.79 \\
\hline Duration of marriage & 3.98 & 2.60 \\
\hline Duration of treatment & 4.77 & 3.61 \\
\hline
\end{tabular}

Table 4 indicates that a significant mean difference exists among three attachment patterns and scores on perceived social support and mental health problems. Post Hoc analysis further revealed that women with secure attachment perceived more social support and faced fewer mental health problems as compared to ambivalent and avoidant attachment pattern.

The following data consists of the linear regression model and subsequent assumptions were met before running the model. The independent and dependent variables are measured at a continuous level and linear relationship exists between two variables, confirmed using SPSS. There is independence of observations, checked using Durbin-Watson statistic in SPSS.

Table 3: Interfactor correlations between perceived social support and mental health problems.

\begin{tabular}{|lllll|}
\hline Factors & Family & Friends & Significant others & DASS-T \\
\hline Family & --- & $0.39 * * *$ & $0.63 * * *$ & $-0.49 * * *$ \\
\hline Friends & --- & --- & $0.41 * * *$ & $-0.31 * * *$ \\
\hline Significant others & --- & --- & --- & $-0.43 * * *$ \\
\hline DASS-T & --- & --- & --- & --- \\
\hline M & 5.93 & 4.11 & 4.69 & 41.39 \\
\hline SD & 2.19 & 1.89 & 1.97 & 10.41 \\
\hline
\end{tabular}

$\mathrm{df}=168, * * * \mathrm{p}<0.001$.

Table 4: One-way analysis of variance among attachment patterns, perceived social support, and with DASS total score.

\begin{tabular}{llllllll|}
\multirow{2}{*}{ Attachment Styles } & \multicolumn{3}{l}{ Secure } & \multicolumn{3}{c}{ Ambivalent } & \multicolumn{3}{c|}{ Avoidant } \\
\cline { 2 - 9 } & M & SD & M & SD & M & SD & F \\
\hline Perceived Social Support & 49.56 & 9.89 & 38.26 & 9.63 & 41.77 & 8.11 & $6.09 * * *$ \\
\hline DASS-T & 42.19 & 10.92 & 47.43 & 9.47 & 44.91 & 8.93 & $4.97 * * *$ \\
\hline
\end{tabular}

Within group, $\mathrm{df}=2$, between group, $\mathrm{df}=166$, total $\mathrm{df}=168 * * * \mathrm{p}<0.001$

Table 5: Model summary.

\begin{tabular}{|lllll|}
\hline & $\mathbf{R}$ & $\mathbf{R}^{2}$ & Adjusted $^{2}$ & Std. error of the estimate \\
\hline Model Summary & $0.690^{\mathrm{a}}$ & 0.748 & 0.315 & 9.14689 \\
\hline
\end{tabular}

a. Predictors: Social Support, Education, Family System, AQA, Husband Education, Age, Years of Treatment, Years of Marriage, b. Dependent Variable: DASS. 
The data show homoscedasticity, so variances along the line of best fit remain uniform as moving up the line. Finally, the regression line's errors are roughly normally distributed. Table 5 lists the $\mathrm{R}$ and $\mathrm{R} 2$ values. The $\mathrm{R}$ value, which highlights the strength of the relationship between two variables, is 0.690 . The $\mathrm{R} 2$ value, which tells how well the data fits the linear regression line, is 0.748 , which is very large.

Table 6: Analysis of variance (ANOVA) between independent variables (social Support, education, family system, AQA, husband education, age, years of treatment, and years of marriage) and the dependent variable (mental health represented by DASS total).

\begin{tabular}{|llllll|}
\hline & Sum of squares & df & Mean Square & F & Sig. \\
\hline Regression & 7140.572 & 8 & 892.571 & 10.668 & $0.000^{\text {a }}$ \\
\hline Residual & 13386.481 & 160 & 83.666 & & \\
\hline Total & 20527.053 & 168 & & & \\
\hline
\end{tabular}

a. Predictors: Social Support, Education, Family System, AQA, Husband Education, Age, Years of Treatment, Years of Marriage, b. Dependent Variable: DASS.

Table 7: The coefficients table.

\begin{tabular}{|c|c|c|c|c|c|c|c|}
\hline & \multicolumn{2}{|c|}{$\begin{array}{l}\text { Unstandardized } \\
\text { coefficients }\end{array}$} & \multicolumn{3}{|c|}{$\begin{array}{l}\text { Standardized } \\
\text { coefficients }\end{array}$} & \multicolumn{2}{|c|}{$95.0 \%$ Confidence Interval for B } \\
\hline & B & Std. Error & Beta & $\mathbf{t}$ & Sig. & Lower bound & Upper bound \\
\hline (Constant) & 48.250 & 7.463 & & 6.466 & 0.000 & 33.512 & 62.988 \\
\hline Age & 0.164 & .230 & 0.067 & 0.712 & 0.043 & -0.291 & 0.619 \\
\hline Education & -2.815 & .595 & -0.336 & -4.734 & 0.000 & -3.990 & -1.641 \\
\hline $\begin{array}{l}\text { Husband } \\
\text { education }\end{array}$ & -0.123 & .573 & -0.015 & -0.214 & 0.831 & -1.255 & 1.009 \\
\hline Family system & -4.488 & 1.954 & -0.160 & -2.297 & 0.023 & -8.347 & -0.629 \\
\hline $\begin{array}{l}\text { Years of } \\
\text { marriage }\end{array}$ & 0.799 & .349 & 0.341 & 2.285 & 0.024 & 0.108 & 1.489 \\
\hline $\begin{array}{l}\text { Years of } \\
\text { treatment }\end{array}$ & 0.028 & .451 & 0.009 & 0.063 & 0.950 & -0.863 & 0.919 \\
\hline AQA & 0.038 & 1.026 & 0.003 & 4.567 & 0.030 & -1.989 & 2.065 \\
\hline Social support & -0.070 & .051 & -0.094 & -1.369 & 0.017 & -0.170 & 0.031 \\
\hline
\end{tabular}

a. Dependent Variable: DASS.

The ANOVA, Table 6 displays how regression equation predicts the independent variable. Table 6 shows that the regression model significantly predicts the dependent variable. The $p$-value of the regression model $(0.000)$ is less than 0.05 , so this model statistically significantly predicts the outcome variable.

Table 7 provides the necessary information to predict DASS from age, education, husband's education, family system, years of marriage, years of treatment, AQA, and social support, as well as establish whether DASS is statistically significant in the model. According to the linear regression model, shown by the "Sig." column, only female's age, education, family system, years of marriage, AQA, and social support are predictors of DASS. Furthermore, the "t" column shows that patient's age and years of marriage positively correlate with DASS, so the older the female is and the longer she has been married, the more mental health problems she would suffer from caused by infertility. On the other hand, the higher the education level of a female, the lower the DASS score. In fact, education is one of the strongest predictors of how likely infertility is to cause mental health issues. Attachment style pattern (AQA) is another strong indicator of DASS score, with secure attachment pattern leading to lowest DASS scores. Moreover, the greater the social support perceived by women, the lower the DASS score.

\section{DISCUSSION}

Infertility is primarily considered a physical concern, but it also affects psycho-social and emotional functioning of an individual. The psychological burden of infertility may lead to many negative outcomes, including anxiety, depression, and loneliness. ${ }^{21}$ The literature review also revealed that women with infertility suffer from more psychological issues compared to men. ${ }^{22}$ This study is anticipated to examine the relationship between attachment pattern, perceived social support, and mental 
health problems in women with primary infertility in the collectivistic and traditional religious context of Pakistan. In traditional collectivistic cultures, women's reproductive health is linked to both their value in the family and society as a whole. ${ }^{18}$ The data was gathered from hospitals and fertility centres in an urbanized city of Pakistan with minimal non-respondent rate. Among other difficulties, it was a challenge to carry out face-to-face interviews, partially due to the hospital environment and the presence of participants' relatives.

The primary findings of this research study signify that an inverse relationship exists between perceived social support and mental health problems, as highlighted by Table 7. These findings are compatible with the past studies showing that social support is a significant factor in bearing the burden of infertility. ${ }^{17,19}$ The reason behind this inverse relationship is that since women get all the blame, they are victimized by emotional and social isolation. No one is there for those females to share the burden of childlessness that may increase the vulnerability of serious mental health concerns. Due to consistent family pressure and negative conditioning by their mothers, men also start to believe that their wife is responsible for the inability to bear a child, which ruins the marital life of such couples. In many cases, the males start thinking of a second marriage since many traditional cultures around the world still permit the practice of polygamy. In a traditional collectivistic culture, where the survival of married life and one's value in society is based on bearing a child, if a woman is unable to fulfill her traditional role, she might experience rejection, isolation, and emotional abuse. Contrarily, if an unfertile woman is supported by her family and most importantly her spouse, showing a secure attachment pattern, then she is less prone to serious mental health issues and depression. This confirms that family is the ultimate unit where an individual feels safe and secure. If an individual perceives less familial support and restricted opportunity to disclose the stress of infertility, then that person faces greater chances of mental health problems as shown in Table 4.

As hypothesized, attachment style is the strongest predictor of mental health of infertile women. Women indicating a secure attachment pattern with their spouses score the lowest on Depression Anxiety Stress Scale as stated in Table 4. It is also interesting that women with primary infertility rate high on ambivalent attachment style (47\%). Unfortunately, there is no comparative data to see the cross-cultural difference in the attachment pattern among women with infertility. Yet, studies have shown that ambivalence attachment may be associated with greater mental health problems, including separation anxiety and depression. ${ }^{26}$ The findings of this study indicate that women with primary infertility scored themselves as ambivalently attached as their specific ambivalent attachment styles show less social support hence more mental health problems. One possible reason may be that a woman with infertility constantly fears her husband opting for a second marriage or giving her a divorce, which would stain her character in society forever. Consequently, this may lead to the development of inconsistent pattern of attachment. This results in greater prevalence of symptoms of anxiety and depression. As studies have indicated, a sense of security, expectations of availability of support system, and responsiveness decreases stress in individuals, and secure attachment lays a role of protective factor against trauma and stress. ${ }^{27}$

Another interesting revelation made by this study is that education is one of the strongest shields against mental health deterioration resulting from infertility (Table 7). Education makes a woman feel independent, strong, and less vulnerable to unjustified societal pressures.28 Indeed, every girl should be encouraged to receive higher education throughout the world so that she can be prepared to stand up for her rights and boldly face the challenges that come later. In many traditional societies to date, females' main role in the family is to bear children and be a homemaker. So, when a woman fails to fulfil one of those roles, she is deemed useless and worthless by the society. On the other hand, professional women contribute financially to the family's well-being and stay productive, which automatically increases their value. $^{29}$

How long a woman has been married and her age also affect how drastically infertility affects her mental health. Older women feel more depressed owing to the fact that they have been waiting for a longer time to conceive, so eventually, they start losing hope and start seeing their infertility as a permanent hurdle in their life. Similarly, the longer a woman has been married, the longer she has been trying to conceive unsuccessfully. Overtime, the stress and pressure from spouse and society increases, so the mental health declines.

This study does have some limitations. The sample size was too modest to make any wide-scale assumptions, so further trials with larger participant pools must be done to see if the same results can be obtained. In addition, the sample was cross-sectional in nature, but the data collection was limited to one city, restricting the generalizability of these findings. Future research should include both rural and urban samples with different psychological variables to find the similarities and differences between various groups of people with diverse backgrounds.

In summary, this study clearly demonstrates that social support and a perception of security in interpersonal relationships plays a role of protective factor against the stress of infertility. It can also be safely concluded that infertility treatment plan should also include health counselling and psycho-education of the family regarding the stress associated with infertility so that negative consequences can be avoided. 


\section{ACKNOWLEDGMENTS}

Authors would like to thank Professor Keith D Renshaw, Chair and Associate Professor, Department of Clinical Psychology at George Mason University, for guidance, encouragement and resourceful critiques on this research work and for his advice and assistance in keeping our progress on schedule, George Mason University and University of Management and Technology for partnering and collaborating together in clinical psychology research to carry out this study.

Funding: No funding sources

Conflict of interest: None declared

Ethical approval: Not required

\section{REFERENCES}

1. World Health Organization (WHO). Progress report in reproductive health research No. 23, 2003.

2. Mascarenhas MN, Flaxman SR, Boerma $\mathrm{T}$, Vanderpoel S, Stevens GA. National, regional, and global trends in infertility prevalence since 1990: a systematic analysis of 277 health surveys. PLoS Medicine. 2012;9(12):1-11.

3. Boivin J, Bunting L, Collins JA, Nygren KG. International estimates of infertility prevalence and treatment-seeking: potential need and demand for infertility medical care. Human Reproduction. 2007;22(6):1506-12.

4. Schmidt, L. Infertility and assisted reproduction in Denmark. Epidemiology and psychosocial consequences. Danish Med Bull. 2006;53(4):390417.

5. Karaca A, Unsal G. Psychosocial problems and coping strategies among Turkish women with infertility. Asian Nursing Res. 2015;9:243-250.

6. Dyer SJ, Abrahams N, Mokoena NE, Spuy ZM. 'You are a man because you have children': experiences, reproductive health knowledge and treatment-seeking behaviour among men suffering from couple infertility in South Africa. Human Reproduction. 2004;19(4):960-7.

7. Remennick L. Childless in the land of imperative motherhood: stigma and coping among infertile Israeli women. Sex role. 2000;11:821-41.

8. Abbasi S, Kousar R, Sadiq SS. Depression and anxiety in Pakistani infertile women. J Surg Pak. 2016;21(1):13-17.

9. Sami N, Ali TS. Psycho-social consequences of secondary infertility in Karachi. J Pak Med Assoc. 2006;56(1):19-22.

10. Bowlby J. Separation, Anxiety, and Anger. Basic Books. 1973;2(1):3-69.

11. Pietromonaco PR, Uchino B, Dunkel S. Close relationship processes and health: implications of attachment theory for health and disease. Heal Psychol. 2013;32(5):499-513.

12. Russell VM, Baker LR, McNulty JK. Attachment insecurity and infidelity in marriage: do studies of dating relationships really inform us about marriage?. J Fam Psychol. 2013;27(2):242-51.

13. Bayley TM, Slade P, Lashen H. Relationships between attachment, appraisal, coping and adjustment in men and women experiencing infertility concerns. Human Reproduction. 2009;24: 2827-37.

14. Donarelli Z, Coco GL, Gullo S, Marino A, Volpes A, Allegra A. Are attachment dimensions associated with infertility-related stress in couples undergoing their first IVF treatment? A study on the individual and cross-partner effect. Human Reproduction. 2012;27(11):3215-25.

15. Walen HR, Lachman ME. Social support and strain from partner, family, and friends: Costs and benefits for men and women in adulthood. J Social Personal Relationships. 2000;17(1):5-30.

16. Decker CL. Social support and adolescent cancer survivors: a review of the literature. Psychooncol. 2007;16:1-11.

17. Martins MV, Peterson BD, Almeida VM, Costa ME. Direct and indirect effects of perceived social support on women's infertility-related stress. Human Reproduction. 2011;26:2113-21.

18. Qadir F, Khalid A, Medhin G. Social support, marital adjustment, and psychological distress among women with primary infertility in Pakistan. Women Heal. 2015;55(4):432-46.

19. Schmidt L, Holstein BE, Christensen U, Boivin J. Communication and coping as predictors of fertility problem stress: cohort study of 816 participants who did not achieve a delivery after 12 months of fertility treatment. Human Reproduction. 2005;20(11):324856.

20. Azghdy HSB, Simbar M, Vedadhir A. The emotional-psychological consequences of infertility among infertile women seeking treatment: Results of a qualitative study. Iranain $\mathbf{J}$ Reproductive Med. 2014;12:131-138.

21. Wiweko B, Anggraheni U, Elvira SD, Lubis HP. Distribution of stress level among infertility patients. Middle East Fertility Soc J. 2017;22:145-8.

22. Greil AL, Blevins SK, McQuillan J. The experience of infertility: A review of recent literature. Sociol Heal Illness. 2010;32(1):140-62.

23. Volgsten H, Svanberg AS, Olsson P. Unresolved grief in women and men in Sweden three years after undergoing unsuccessful in vitro fertilization treatment. Acta obstetricia et gynecologica Scandinavica. 2010;89(10):1290-7.

24. Zimet GD, Dahlem NW, Zimet SG, Farley GK. The Multidimensional Scale of Perceived Social Support. J Personality Assessment. 1988;52(1):30-41.

25. Lovibond SH, Lovibond PF. Manual for the Depression Anxiety Stress Scales. 2nd ed. Sydney: Psychology Foundation; 1995.

26. Mofrad S, Abdullah R, Uba R. Attachment patterns and separation anxiety symptom. Asian Social Science. 2010;6(11):148-53. 
27. Dallaire DH, Weinraub M. Predicting children's separation anxiety at age 6: The contributions of infant-mother attachment security, maternal sensitivity, and maternal separation anxiety. Attachment Human Development. 2005;7(4):393408.

28. Hazan C, Shaver P. Romantic love conceptualized as an attachment process. J Personality Social Psychol. 1987;52(3):511.

29. Yazdani F, Kazemi A, Fooladi MM, Samani HR. The relations between marital quality, social support, social acceptance and coping strategies among the infertile Iranian couples. Europ J Obstetrics Gynecol Reproductive Biol. 2016;200:58-62.

Cite this article as: Saleem S, Qureshi NS,

Mahmood Z. Attachment, perceived social support and mental health problems in women with primary infertility. Int J Reprod Contracept Obstet Gynecol 2019;8:2533-40. 